\title{
An Online Distributed Game Optimal Control for Heavy Haul Trains with Limited Communication
}

\author{
Kai Gao, Zhiwu Huang, Jun Peng, Heng Li, and Weirong Liu \\ School of Information Science and Engineering, Central South University, Changsha, Hunan 410075, China \\ Correspondence should be addressed to Zhiwu Huang; hzw@csu.edu.cn
}

Received 11 August 2014; Accepted 23 October 2014

Academic Editor: Guangming Xie

Copyright (C) 2015 Kai Gao et al. This is an open access article distributed under the Creative Commons Attribution License, which permits unrestricted use, distribution, and reproduction in any medium, provided the original work is properly cited.

For heavy haul trains, it is difficult to get global information due to the limited range of communication. This paper proposed a novel distributed optimal control based on game strategy, in which the global optimization is achieved by equilibrizing subsystems' performance just utilizing local information. To online solve the game control, an efficient multivariable extremum seeking algorithm was adapted to approximate the partial differential equation deduced by optimal condition. The convergence of the proposed approximate algorithm is proved by constructing a fictitious Lie bracket system using Lyapunov function. Finally, the proposed distributed optimal control is valuated rigorously by case study according to the configuration of Daqin railway in China.

\section{Introduction}

Heavy haul trains are used broadly in many countries with high demand for transporting mineral, petroleum, coals, and so on. Essentially, heavy haul trains are distributed powered networked system constituted with many locomotives and wagons. The basic control problem for heavy haul trains is tracking to the target speed profile while considering some performance index, including the traveling time, energy consumption, and in-train forces. Then, the optimal controller should be designed to achieve the performance.

The optimal problem has received many attentions recently in the field of train's operating control [1-5]. Energy consumption and traveling time focused optimal control was studied in these works for either passenger trains or ordinary freight trains. But much larger dynamic in-train forces were imposed in heavy haul trains due to unreasonable control of the distributed power, undulating grades, and also the lager train lengths. The couplings used in heavy haul trains wear out due to large in-train forces [1]. So the optimization techniques used for passenger trains or ordinary freight trains may not be directly applicable to heavy haul trains.

Researches on optimal control of heavy haul trains were done about travelling time, in-train forces, and energy consumption. An optimal open-loop offline controller is designed for cruise control of heavy haul trains [6]. It is derived that distributed controller can be more useful to minimize the in-train forces. But static error always exists for the reason of open loop. Based on this, the authors did further research on closed-loop optimal controller of heavy haul trains with consideration of in-train forces, speed tracking error, and energy consumption. It concluded that the 2-2 mode (full distributed) controller's performance about intrain forces is much better than 1-1 mode (centralized). A similar conclusion was derived in [7]. In these literatures, a LQR (linear quadratic regulator) optimal method is used to optimize the feedback gain using global information [6, 7]. The global information is gathered by communication networks laid on the train, for example, Lonworks (used in electronically controlled pneumatic (ECP)), GSM-R, or LTE$\mathrm{R}$. But the global information is not always available during the traveling due to the range of communication, disturbance, delay, and communication failure. Therefore, design of a distributed optimal controller for each powered locomotive and wagon is more practical and intriguing using available local information about the whole system.

Local performance index should be constructed first when designing distributed optimal controllers for heavy haul trains. The subsystems in the train work in a team to track a desired speed profile. At the same time, each of 
them tries to minimize the energy consumption and in-train forces at the cost of increasing others, which is very complex and difficult. Game strategy is an efficient method to solve these types of problems with conflict benefits [8]. Distributed optimal control problem was solved by game strategy for multiagent system in some works [9-11].

In [9], a noncooperative game was designed to distributed optimization with fixed local communication. The authors design estimators to estimate other players' states which make the controller compute heavily. Since there is information exchange among the players, there is no need to design so many estimators. A most related work is done in [10], which brings together cooperative control, reinforcement learning, and game theory to solve multiplayer differential games on communication graph topologies. It formulates graphical games for dynamic systems and provides policy iteration and learning algorithms along with proof of convergence to the Nash equilibrium. Since there are communications, the subsystems cooperate to track the target speed.

A differential game in the sense of cooperation is constructed in [12] and Pareto equilibrium is further studied for cooperative game [13]. Analysis of convergence to Pareto equilibrium was done and the necessary and sufficient conditions were derived. It showed that if the dynamic system is controllable then all Pareto candidates can be obtained by solving the necessary conditions of a weighted sum optimal control problem. However, this is done offline and global information is required.

In this paper, we made an improvement on these works. We propose a novel distributed optimal control based on game strategy, in which the global optimization is achieved by equilibrizing subsystems' performance just utilizing local information. To online solve the game, an efficient multivariable extremum seeking algorithm was adapted to approximate the partial differential equation deduced by optimality condition. The proposed controller is applied to heavy haul trains with second order interconnected model.

The main contribution of this paper includes four aspects. Firstly, a distributed optimal controller using local information with conflict performance index is proposed for heavy haul trains with a second order interconnected model. A system level global target was attained by optimizing the local performance with proper communication topology. Secondly, a cooperative differential game is constructed to solve the distributed optimal problem with conflicting individual objectives. We proved that a Pareto equilibrium would be reached when the communication topology of the subsystem in heavy haul trains was strongly connected. Thirdly, we proved that the cooperative game can be solved only by local information, while, in most existing literatures, global information is necessary for solving the game. Lastly, we develop an efficient multivariable extremum seeking algorithm, which was proposed to approximate the partial differential equation deduced by solving the cooperative game.

The remainder of this paper is organized as follows. In Section 2, the model of heavy haul trains is given, including kinetic model and communication connections model. In Section 3, the distributed optimal control for heavy haul trains is formulated and then a cooperative game is constructed. In Section 4, the stability and convergence of formulated cooperative game are analyzed. In Section 5, a multivariable extremum seeking algorithm is designed to approximate the partial differential equation deduced by solving the cooperative game. In Section 6, a simulation scenario is set based on application. At last, the conclusion is given.

\section{Model of Heavy Haul Trains}

In this section, the formulation of distributed optimal control problem and the train model used in this paper is summarized.

There are two types of model of heavy haul trains discussed in the existing literatures, namely, point-mass model used in $[3,14]$ and spring-mass model used in $[7,15]$. Spring-mass model is mostly used when considering the complex in-train dynamics and the effectiveness is validated against experimental data collected on a train operated by Spoornet on its COALlink in South Africa [16]. The wired Lonworks communication or wireless GSM-R and LTE-R communication is used to operate heavy haul trains more efficiently with the development of communication based train control system. In our paper, the spring-mass model of heavy haul trains is used with considering the communication connections among the cars (for ease of presentation, in what follows both locomotives and wagons in heavy haul trains are referred to as cars), which is more realistic.

2.1. Kinetic Model of Heavy Haul Trains. The longitudinal dynamic characters of heavy haul trains can be described by the following set of equations $[7,16]$ :

$$
\begin{gathered}
\dot{x}_{1}=v_{1}, \\
\dot{v}_{1}=\frac{u_{1}}{m_{1}}-\frac{F_{1}^{C}}{m_{1}}, \\
\dot{x}_{i}=v_{i}, \\
\dot{v}_{i}=\frac{u_{i}}{m_{i}}-\frac{F_{i}^{C}}{m_{i}}+\frac{F_{i-1}^{C}}{m_{i}}, \\
\dot{x}_{n}=v_{n}, \\
\dot{v}_{n}=\frac{u_{n}}{m_{n}}+\frac{F_{n-1}^{C}}{m_{n}},
\end{gathered}
$$

where $i=2, \ldots, n-1$ and $n$ (positive integer) is the number of cars in heavy haul trains. When $x_{i}, v_{i}$, and $m_{i}$ denote the displacement with respect to an inertial frame, the speed, and the mass of the $i$ th car, respectively, $u_{i}$ is the traction force or braking force added to the $i$ th car. $F_{i}^{C}$ is the in-train force between the $i$ th and $(i+1)$ th car and can be formulated as follows:

$$
F_{i}^{C}=k_{i}\left(x_{i}-x_{i+1}\right)+d_{i}\left(\dot{x}_{i}-\dot{x}_{i+1}\right),
$$

where $k_{i}$ is the coupler's spring constant of the $i$ th car and $d_{i}$ is the coupler's damp constant of the ith car. 


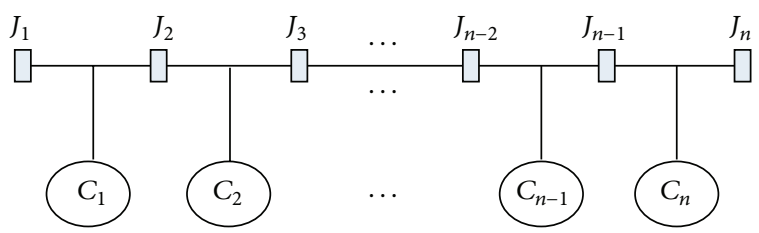

(a) Wired network equipped on heavy haul trains
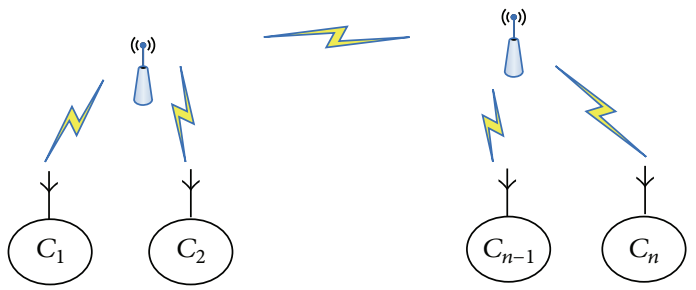

(b) Wireless network equipped on heavy haul trains

Figure 1: Two types of network equipped on heavy haul trains. In (a) $C_{i}$ ( $i \in n, n$ is the number of cars in the train) is the $i$ th controller equipped on the cars. They can communicate with each other by wired Lonworks protocol. $J_{i}$ is the conjunction box between the cars. (b) It is a wireless network configuration of heavy haul trains. The physical connections are ignored in the figure.

The in-train forces can be viewed as the coupling force between the cars and we can rewrite (1) as follows:

$$
\dot{\vec{z}}_{i}=\vec{A} \vec{z}_{i}+\sum_{j=i-1}^{i+1} c_{i j} \Theta \vec{z}_{j}+\vec{B} u_{i}
$$

where the letter $\vec{x}$ is used to denote a vector. $\vec{z}_{i}=\left[\begin{array}{ll}x_{i} & v_{i}\end{array}\right]^{T} \epsilon$ $R^{2}$ is the $i$ th car's states vector, the matrix $\vec{A}=\left[\begin{array}{llll}0 & 1 ; & 0 & 0\end{array}\right]$, the matrix $\vec{B}=\left[\begin{array}{ll}0 & 1\end{array}\right]^{T}, c_{i j}$ is coupling coefficient, and $c_{i j}=1$ when $i \neq j, c_{i j}=-2$ when $i=j, \Theta=\left[\begin{array}{llll}0 & 0 ; & k / m & d / m\end{array}\right]$ is the coupling matrix where we suppose that all the $k_{i}$ and $d_{i}$ are equal, if the types of couplers in the train are the same actually, and $u_{i}$ is the control input.

2.2. Model of Communication Connections among the Cars. As a benefit from the communication network equipment laid on heavy haul trains, more flexible control strategy can be designed. Lonworks is the most popular network protocol used in heavy haul trains equipped with $\mathrm{ECP} / \mathrm{iDP}$ systems as shown in Figure 1(a). However, heavy haul trains need to marshal when loading and unloading freight, and then the wireless network equipped on the train will be more flexible, such as LTE-R (Long Term Evolution-Railway), as shown in Figure 1(b).

Remark 1. It is obvious that there are some drawbacks of configuration as shown in Figure 1(a). If one of the nodes fails, the whole network will be broken. Moreover, frequent marshalling of the train makes it difficult and expensive to maintain. Configuration (b) is much more flexible and can provide larger bandwidth. LTE-R has already been laid out in Shuohuang Railway in China.

A direction graph is used to describe the communication among subsystems. According to the trains model equation (1), there are $n$ vertexes $v=\left\{v_{1}, v_{2}, \ldots, v_{n}\right\}$ in the Graph $\mathscr{G}(v, \varepsilon, \mathscr{A})$, which is used to describe the communication among the cars (a node in the graph) similarly as [17]. The set of edges or arcs is $\varepsilon \subseteq v \times v$. An edge from node $j$ to node $i$ is denoted by $\left(v_{j}, v_{i}\right)$, which means that node $i$ receives the information from node $j$. The associated adjacency matrix $\mathscr{A}=\left[a_{i j}\right] \in R^{n \times n}$, where $a_{i j}=1$ if $\left(v_{j}, v_{i}\right) \in \varepsilon$, means node $i$ can receive information from node $j, a_{i j}=0$ otherwise. Particulalry, $a_{i i}=0$; that is to say, there is no self-loop in the Graph. Node $j$ is called a neighbor of node $i$ if $\left(v_{j}, v_{i}\right) \in \varepsilon$. The set of neighbors of node $i$ is denoted as $N_{i}=\left\{v_{j} \in v:\left(v_{j}, v_{i}\right) \in \varepsilon\right\}$. The degree of node $i$ is defined as $d_{i}=\sum_{j \in N_{i}} a_{i j} . N \backslash\{i\}$ is used to denote all of the cars except $i$. If there is a sequence of nodes $v_{1}, v_{2}, \ldots, v_{j}$ such that $\left(v_{i}, v_{i+1}\right) \in \varepsilon, i \in\{1,2, \ldots, j-1\}$, then there is a path from $v_{1}$ to $v_{j}$. A direction graph is said to have a spanning tree, if there is a node $i_{r}$ (called the root), such that there is a directed path from the root to every other node in the graph. In the communication graph $\mathscr{G}$, if there is a path from node $i$ to node $j$, we denote that $j$ is reachable from $i$. If there is at least a path between any two nodes $i$ and $j$, the graph is strongly connected [18].

2.3. Desired Speed Profile. The heavy haul trains travel according to a desired speed profile and the speed profile can be expressed as

$$
\dot{x}_{0}=v_{0}, \quad \dot{v}_{0}=u_{0}
$$

where $x_{0}, v_{0}$, and $u_{0}$ are the desired displacement, speed, and input, respectively, and $\vec{z}_{0}=\left[\begin{array}{ll}x_{0} & v_{0}\end{array}\right]^{T}$.

Usually, we can view the desired speed profile as the operator's command and can be scheduled offline due to experience or online heuristic or close-loop global optimality of energy consumption, traveling time, and so on. However how to design the desired speed profile is not our focus in this paper. If the desired speed profile is scheduled appropriately, and the heavy haul trains can track the profile well under our proposed controller, the trains can travel with favorable performance.

\section{Control Problem Formulation}

3.1. Distributed Optimal Control with Neighbor's Information. Due to the different control requirements caused by undulating grades of the railway and unpredictable communication failure among cars in the train, distributed controllers are designed by neighbor's information (available information from cars in the train, and global information is not necessary), with considering some performance index to track 
the desired speed profile. The disagreement of car $i$ to his neighbors and the desired speed profile is denoted in

$$
\vec{\eta}_{i}=\sum_{j \in N_{i}} a_{i j}\left(\vec{z}_{i}-\vec{z}_{j}\right)+l_{i 0}\left(\vec{z}_{i}-\vec{z}_{0}\right)
$$

where $a_{i j}$ is defined in the section of communication model and $l_{i 0}=1$ if the car $i$ can directly receive the operator's command.

The controllers in the train cooperate to achieve a system level objective. So, the objective of our work is to design $u_{i}$ in (1) with optimizing the local performance index $J_{i}$ as designed in

$$
\begin{aligned}
J_{i} & =\int_{0}^{\infty} L_{i}\left(\vec{\eta}_{i}, u_{i}, t\right) d t \\
& =\int_{0}^{\infty}\left(\vec{\eta}_{i} Q_{i i} \vec{\eta}_{i}^{T}+u_{i} \vec{R}_{i i} u_{i}^{T}+\sum_{j \in N_{i}} u_{j} \vec{R}_{i j} u_{j}^{T}\right) d t
\end{aligned}
$$

where $Q_{i i}, \vec{R}_{i i}$, and $\vec{R}_{i j}$ are weight coefficients. In the performance index $J_{i}, Q_{i i}$ is the weight of tracking desired speed and the in-train forces and $\vec{R}_{i i}$ is the weight of energy consumption and $\vec{R}_{i j}$ is the weight of energy consumption of neighbor cars. To minimize the performance index $J_{i}$ means optimization about the train's travel time, in-train forces, and energy consumption [7]. The significant difference is $J_{i}$ defined in (6) depending only on local neighbors' information and the performance index may be coupled and conflict. We can see that decreasing the input of one car will result in increasing inputs of others, which makes solving of optimal control problem very difficult. The distributed optimal control problem discussed in this paper will be formulated as a cooperative game problem, which is capable of solving problems of many individuals with cooperative and/or conflict interests.

\subsection{Applying Cooperative Game Strategy to Heavy Haul} Trains. As discussed in the section of distributed optimal control problem, it is converted to a cooperative game. The detailed description and definition will be given as follows.

A four-tuple $\left[\begin{array}{llll}\vec{z}_{i} & \phi_{i} & u_{i} & J_{i}\end{array}\right]$ is used to describe the state of a car in the train. $u_{i}$ is a controller's decision value, namely, control input, considering the neighbors' states. $u_{-i}$ denotes the decision values of $i$ 's neighbors. $\boldsymbol{U}_{i}$ is used to denote the set of available decisions for car $i$. A tuple $\vec{u}=$ $\left(u_{1}, u_{2}, \ldots, u_{n}\right)=\Pi_{i} \mathscr{U}_{i} \in \mathcal{U}$ is denoted as a joint value. A function $V_{i}\left(\eta_{i}(t)\right)$ is used to denote the cost of taking decision $u_{i}$. The cost function is defined as (7), which coincides with performance index $J_{i}$ :

$$
V_{i}\left(\vec{\eta}_{i}(t)\right)=\int_{t}^{\infty}\left(\vec{\eta}_{i} Q_{i i} \vec{\eta}_{i}^{T}+u_{i} R_{i i} u_{i}^{T}+\sum_{j \in N_{i}} u_{j} R_{i j} u_{j}^{T}\right) d t .
$$

Assumption 2. Assume that $V_{i}\left(\vec{\eta}_{i}(t)\right)$ is continuous and firstorder derivative and from the expression equation (7), it is an integral of quadratic form function, then $V_{i}$ is nonnegative.

The neighbors' states are available to make a decision for car $i$. It is always impossible to minimize the performance index for a single car without increasing that of other cars. It implies that there exists an equilibrium for the whole system, which coincides with the characters of Pareto optimality. The Pareto optimality equilibrium for cooperative game is defined in [12], with global information of the system.

Lemma 3. Global Pareto optimality: $u^{*}$ are Pareto optimal solutions for the formulated cooperative game, if and only if, for $\forall i \in n, u^{*}$ minimizes $J_{i}(u)$ on the constrained set:

$$
\mathscr{U}_{i} \triangleq\left\{u \in \mathcal{U} \mid J_{j}\left(u^{*}\right) \leq J_{j}(u), \forall j \in N \backslash\{i\}\right\} .
$$

However, when only partial information is available to design the decision during game, Lemma 3 needs to be extended in the sense of local Pareto optimality equilibrium. If the communication topology of the system meets some conditions, the global Pareto optimality equilibrium will be reached on the basis of local Pareto optimality equilibrium. The definition of local Pareto optimality equilibrium is given in Definition 4.

Definition 4. Local Pareto optimality: subsystem i's Pareto best response to neighbours' fixed $u_{-i}, i \in N_{i}$ is the rule $u_{i}^{*}$ such that minimizing $J_{i}$ and for all $j \in N_{i}$, the following inequation holds, $J_{j}\left(u_{i}^{*}, u_{-i}^{*}\right) \leq J_{j}\left(u_{i}, u_{-i}\right)$.

The objective of the paper is to design a decision making algorithm while playing the cooperative game. The game converges to a global Pareto optimality when every player is achieving local Pareto optimality.

For a distributed optimal problem formulated in our paper, if the designed decision making rule $u_{i}, \forall i$ to play the game is continuous and $u_{i}(0)=0$ can stabilize the disagreement $\vec{\eta}_{i}$ of every car locally with minimizing $J_{i}$, and the value function $V_{i}\left(\vec{\eta}_{i}(t)\right)$ is finite, we say that a global optimization is reached under distributed optimal controllers by cooperative game.

Design $u_{i}$ to minimize the value function $V_{i}\left(\vec{\eta}_{i}(t)\right)$ subject to the state transition function of the system equation (3) and the initial states $\vec{z}_{i}(0)$ with states bound $\underline{\gamma}_{i} \leq u_{i} \leq \bar{\gamma}_{i}$.

By Lemma 3 and conclusion from [13], this game can be solved by a group of constrained optimal equations. Construct a Hamiltonian function with boundary conditions and $\underline{\gamma}_{i} \leq u_{i} \leq \bar{\gamma}_{i}$ as

$$
\begin{aligned}
H_{i}\left(u_{i}, u_{-i}, \vec{\eta}_{i}, \vec{z}_{0}\right)= & \xi_{i}(t) \phi_{i}\left(\vec{z}_{i}, \vec{z}_{j}, u_{i}\right)+L_{i}+u_{j} \vec{R}_{j j} u_{j}^{T} \\
& +\zeta_{i}\left(u_{i}-\bar{\gamma}_{i}\right)+\kappa_{i}\left(\underline{\gamma}_{i}-u_{i}\right),
\end{aligned}
$$

where $\phi_{i}\left(\vec{z}_{i}, \vec{z}_{j}, u_{i}\right)=\dot{\vec{z}}_{i}$ and $\dot{\vec{z}}_{i}$ is denoted in (3), $L_{i}$ is defined in (6), and $\zeta_{i}$ and $\kappa_{i}$ are positive real numbers [19]. To minimize the local performance index equation (6) with 
constrains, by Pontryagin's minimum principle and KarushKuhn-Tucker condition, the following equations should be satisfied:

$$
\begin{gathered}
\frac{d H_{i}(\cdot)}{d t}=0, \\
\dot{\vec{z}}_{i}^{*}=\phi_{i}\left(\vec{z}_{i}^{*}\right), \\
\dot{\xi}_{i}(t)=-\frac{\partial H_{i}}{\partial \vec{\eta}_{i}}, \\
\frac{\partial H_{i}}{\partial u_{i}}=0, \\
\vec{z}(0)=\vec{z}_{0},
\end{gathered}
$$

where

$$
\begin{gathered}
\dot{\xi}_{i}(t)=-\vec{A} \xi_{i}(t)-2 \vec{Q}_{i i} \vec{\eta}_{i} \\
\frac{\partial H_{i}}{\partial u_{i}}=\xi_{i}(t)\left(d_{i}+l_{i 0}\right) \vec{B}^{T}+2 u_{i} \vec{R}_{i i}-\kappa_{i}+\zeta_{i} \\
\Longrightarrow u_{i} \\
=-\frac{1}{2}\left(\xi_{i}(t)\left(d_{i}+l_{i 0}\right) \vec{R}_{i i}^{-1} \vec{B}^{T}-\kappa_{i} \vec{R}_{i i}^{-1} \overrightarrow{1}_{2}+\zeta_{i} \vec{R}_{i i}^{-1} \overrightarrow{1}_{2}\right)
\end{gathered}
$$

if $\kappa_{i}=\zeta_{i},(12)$ can be written as

$$
u_{i}=-\frac{1}{2} \xi_{i}(t)\left(d_{i}+l_{i 0}\right) \vec{R}_{i i}^{-1} \vec{B}^{T} .
$$

\section{Stability and Convergence of Cooperative Game}

Theorem 5. Let car $i$ in the heavy haul trains be reachable for all $i \in n$, and let every car $i$ be in local Pareto optimality as Definition 4 to all of its neighbors $j \in N_{i}$. Then all the cars are in global Pareto optimality as Lemma 3 if and only if the graph is strongly connected.

Proof. If every car $i$ is in local Pareto optimality to its neighbours $j \in N_{i}$, then follow the Definition 4, we have $\forall i$, and $J_{i}$ is minimized at $J_{j}\left(u_{i}, u_{-i}\right) \leq J_{j}\left(u_{i}^{*}, u_{-i}^{*}\right)$, where $j \in N_{i}$. We analyze the problem from a viewpoint of propagation. Choose any car labeled $i$, its neighbours are $N_{i}$, and not all the cars are included in this group. Then for this group $\mathrm{Gr}_{i}$, the Pareto optimal is reached. Because the graph is strongly connected, for the group $\mathrm{Gr}_{i}$, there is at least a car $k$ which is reachable for other cars not in group $\mathrm{Gr}_{i}$. It is the same for the group $\mathrm{Gr}_{k}$ which is different from $\mathrm{Gr}_{i}$. Then the Pareto optimality is propagated from $\mathrm{Gr}_{i}$ to $\mathrm{Gr}_{k}$. The Pareto optimality is propagated to other cars until there is no any car left in the graph. Then we can say that the global Pareto optimality is reached.

Necessity. If the graph is not strongly connected, there is at least a pair of $i$ and $j$ between which there is no path. Then the Pareto optimalilty related with $i$ cann't propagate to $j$, and then the global Pareto optimality is not achieved.

Sufficiency. When the graph is strongly connected, the global Pareto optimality is not achieved and only some local Pareto optimality is achieved, for example, $\mathrm{Gr}_{i}, \mathrm{Gr}_{j}$, and $\mathrm{Gr}_{k}$. That is to say, $i \in N_{i}, i \notin N_{j}$, and $i \notin N_{k}$. By the view of Pareto optimality propagation, we can deduce that there is no path from $i$ to $j$ or $k$, so this is contradictory to the graph that is strongly connected.

Theorem 6 (stability of cooperative distributed optimal controller). Let Assumption 2 hold. To design a distributed cooperative optimal controller through game, according to (13), aiming at effecting on state disagreement and deviation to the desired profile, $u_{i}$ should be related with the variety of $\eta_{i}$, that is $\partial V_{i} / \partial \eta_{i}$. Given $\xi_{i}(t)=\partial V_{i} / \partial \eta_{i}, \forall i$, under the Pareto optimal $u_{i}^{*}$, the heavy haul trains are asymptotically stable and terminally converge to the desired speed profile.

Proof. To analyze the stability of cooperative distributed optimal control, we utilize the Lyapunov function. Choose the value function $V_{i}$ as a Lyapunov function, $V_{i} \geq 0$. The time derivative of $V_{i}$ is as follows:

$$
\begin{aligned}
\dot{V}_{i} & =\frac{\partial V_{i}^{T}}{\partial \vec{\eta}_{i}} \dot{\vec{\eta}}_{i} \\
& =-L_{i}-\sum_{j \in N_{i}}\left(\vec{\eta}_{j} Q_{j j} \vec{\eta}_{j}^{T}+u_{j} \vec{R}_{j j} u_{j}^{T}+\sum_{k \in N_{j}} u_{k} \vec{R}_{j k} u_{k}^{T}\right),
\end{aligned}
$$

where $L_{i}$ is designated in (6). The terms in $\dot{V}_{i}$ are quadratic form, and then we can derive that $\dot{V}_{i}<0$. So the system is asymptotically stable and $\vec{\eta}_{i} \rightarrow 0$. That is to say $\vec{z}_{i} \rightarrow \vec{z}_{j} \rightarrow$ $\vec{z}_{0}$, the states converge to the desired profile.

\section{Multivariable Extremum Seeking Algorithm for Cooperative Game}

To drive the cooperative game to a Pareto optimal equilibrium, the decision rule equation (13) must be computable. According to Theorem $6, u_{i}$ is in the form of

$$
u_{i}=-\frac{1}{2} \frac{\partial V_{i}}{\partial \vec{\eta}_{i}}\left(d_{i}+l_{i 0}\right) \vec{R}_{i i}^{-1} \vec{B}^{T}
$$

Substituting $u_{i}$ into (9), we derive a partial differential equation. It is hard to get an analytical solution for such an equation. Extremum seeking algorithm provides a numerical perturbation based method to steer an unknown dynamical system to the optimality. Motivated by [20, 21], a multivariable extremum seeking estimator is designed to solve the Pareto optimality. A basic multivariable extremum seeking algorithm is given in [22], which is based on perturbation and periodic (sinusoidal) excitation signals are primarily used to probe the nonlinearity and couplings. According to the equations of $u_{i}$ and $\vec{\eta}_{i}$, block diagram of the multivariable extremum seeking algorithm for solving Pareto optimality is given as Figure 2, where $h_{i}$ is a vector to pick $J_{i}$ whose $i$ th element is 1 , and other elements are $0 . \vec{f}_{i}$ is a vector of $\left[1,2\left(d_{i}+l_{i 0}\right)^{-1} \vec{B}^{-1} \vec{R}_{i i}\right]$, and then the estimator of $\left(u_{i}, \vec{\eta}_{i}\right)$ can be 


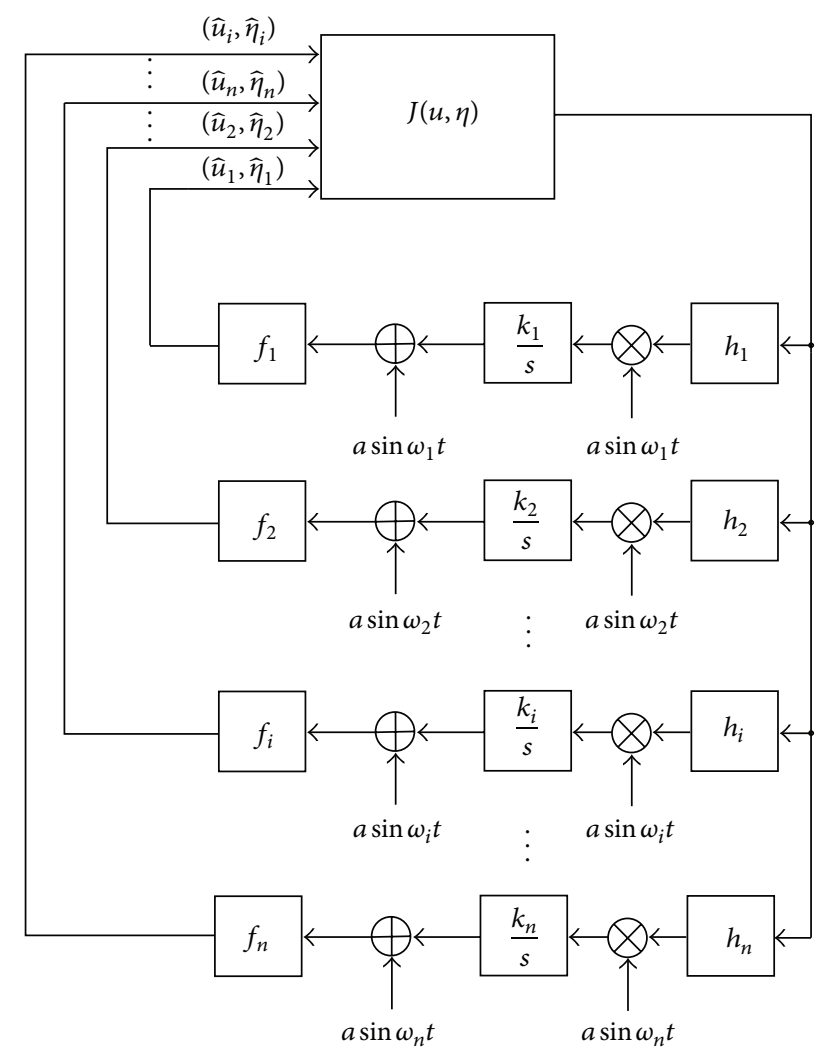

FIGURE 2: Block diagram of multivariable extremum seeking algorithm for solving Pareto optimal.

derived as $\left(\widehat{u}_{i}, \widehat{\vec{\eta}}_{i}\right) . a, \omega_{i}$, and $k_{i}$ are real numbers. According to the extremum seeking algorithm shown as Figure 2, one has

$$
\begin{gathered}
\dot{\vec{u}}_{i}=a k_{i} J_{i} \sin \omega_{i} t+a \omega_{i} \cos \omega_{i} t, \\
\dot{\overrightarrow{\hat{\eta}}}_{i}=a k_{i} \rho_{i} J_{i} \sin \omega_{i} t+a \omega_{i} \rho_{i} \cos \omega_{i} t \\
\rho_{i}=2\left(d_{i}+l_{i 0}\right)^{-1} \vec{B}^{-1} \vec{R}_{i i} .
\end{gathered}
$$

Remark 7. In our designed extremum seeking algorithm, $J_{i}$ is not measured directly but can be derived by measuring other states according to (6), which depends on local available states. $\omega_{i}$ affects the frequency of perturbation and avoiding of reaching a local optimality for nonconvex system. It is insignificant to set $\omega_{i}$ uniformly as $\omega$ for simplify. $k_{i}$ is set similarly. By the multivariable extremum seeking algorithm, we aim at forcing the solutions $\left(\widehat{u}_{i}, \widehat{\vec{\eta}}_{i}\right), \forall i \in n$ finally converge to the Pareto optimal $\widehat{u}_{i}^{*}, \widehat{\vec{\eta}}_{i}^{*}, \forall i \in n$, and then the distributed optimal problem is solved.

Theorem 8. Consider a distributed optimal problem aiming at tracking the desired profile which would be solved by a game, to minimize the performance index $J_{i}, \forall i \in n$ as defined in (6), Lemma 3. Through a multivariable extremum seeking algorithm described in Figure 2 and (16), the Pareto optimality can be solved online at $J_{i}^{*}, u_{i}^{*}$ and $\eta_{i}^{*}$. If $\omega_{i}$ is large sufficiently, the Pareto optimal solution $\left(\widehat{u}_{i}^{*}, \widehat{\vec{\eta}}_{i}^{*}\right), \forall i \in n$, is globally uniformly asymptotically stable for system as described in (16).

Proof. Motivated by [21], a Lie brackets based analysis method is developed. Firstly, we consider the stability of $u_{i}$. According to (16), the overall states can be written as

$$
\vec{u}=\vec{f} v_{1}+\vec{g} v_{2}
$$

where $\vec{u} \quad=\left[\begin{array}{llll}u_{1} & u_{2} & \cdots & u_{n}\end{array}\right]^{T}, \quad \vec{f}=$ $\left[\begin{array}{llll}a k_{1} J_{1} & a k_{2} J_{2} & \cdots & a k_{n} J_{n}\end{array}\right]^{T}$, and $\vec{g}=$ $\left[\begin{array}{llll}a \omega_{1} & a \omega_{2} & \cdots & a \omega_{n}\end{array}\right]^{T} \cdot \nu_{1}=\sin \omega_{i} t$ and $\nu_{2}=\cos \omega_{i} t$ are fictitious control input. Then an input affine system is constructed. follows:

The Lie bracket operation of vector $\vec{f}$ and $\vec{g}$ is defined as

$$
[\vec{f}, \vec{g}]=-a^{2} \sum_{i=1}^{n} k_{i} \omega_{i} \nabla_{u_{i}} J_{i}
$$

where $\nabla_{u_{i}} J_{i}$ is the gradient of $J_{i}$ at the direction of $u_{i}$. Then a Lie bracket system can be defined according to (18):

$$
\dot{\vec{u}}^{\prime}=[\mathbf{0}]+\frac{\psi_{12}}{2 \pi}[\vec{f}, \vec{g}],
$$

where $\psi_{12}=\int_{-\pi}^{\pi} \int_{0}^{\theta} \sin \tau \cos \theta d \tau d \theta$, calculating the integral, and $\psi_{12}=\pi$. Then the Lie bracket system can be written as

$$
\dot{\vec{u}}_{i}^{\prime}=-\frac{1}{2} a^{2} \sum_{i=1}^{n} k_{i} \omega_{i} \nabla_{u_{i}} J_{i}
$$

Construct a global Lyapunov function as $V_{L}=\sum_{i=1}^{n} J_{i}-$ $\sum_{i=1}^{n} J_{i}^{*}$. It is obvious that $V_{L} \geq 0$ because $J_{i}^{*}$ is minimized. The time derivative of $V_{L}$ is as follows:

$$
\dot{V}_{L}=\sum_{i=1}^{n} \nabla_{u_{i}} J_{i} \dot{u}_{i}^{\prime}
$$

Substitute (20) into (21),

$$
\dot{V}_{L}=-\sum_{i=1}^{n}\left[\frac{1}{2} a^{2} \sum_{i=1}^{n} k_{i} \omega_{i}\left(\nabla_{u_{i}} J_{i}\right)^{2}\right] \leq 0
$$

when $V_{L}=0$ and $\dot{V}_{L}=0$, we can see that $J_{i}$ will converge to Pareto optimal equilibrium $J_{i}^{*}$ for all $i$ and the optimal control $u_{i}^{*}$ will be achieved at the same time. So the system is globally uniformly asymptotically stable.

\section{Results and Discussion}

To validate the performance of proposed distributed cooperative optimal controller, a simulation scenario based on field application is set. A segment of track, desired speed profile Figure 3, and simulation parameters of trains are given in Table 1, which comes from heavy haul trains running on Daqin railway in China. Without loss of generality, the number of locomotives of heavy haul trains is set to be four 


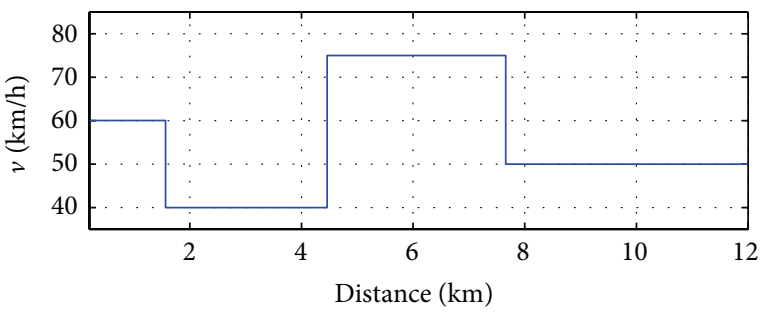

(a)

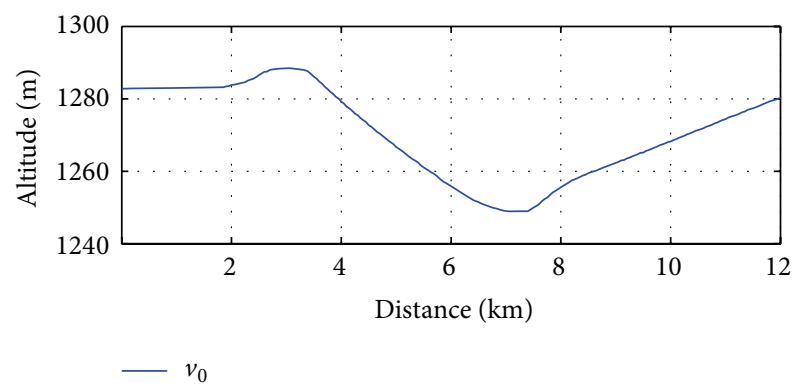

(b)

FIGURE 3: Desired speed profile.

TABLE 1: The simulation parameters.

\begin{tabular}{lcc}
\hline Parameter & Value & Unit \\
\hline Mass $m_{i}$ & 292000 & $\mathrm{~kg}$ \\
Locomotive length & 20 & $\mathrm{~m}$ \\
Coupler length $L_{i, i-1}$ & 1 & $\mathrm{~m}$ \\
$k_{i}$ & $100 \times 10^{6}$ & $\mathrm{Nm}^{-1}$ \\
$d_{i}$ & $100 \times 10^{4}$ & $\mathrm{Nsm}^{-1}$ \\
Max traction & 450 & $\mathrm{kN}$ \\
Max brake & 382 & $\mathrm{kN}$ \\
Number of wagons & 200 & - \\
Number of locomotives & 4 & - \\
Start point & 0.2 & $\mathrm{~km}$ \\
End point & 12 & $\mathrm{~km}$ \\
\hline
\end{tabular}

in the simulation, which is a $1+2+1$ (namely, one locomotive + some wagons + two locomotives + some wagons + one locomotive) mode usually used in Daqin. Traction or braking of the train mainly act on locomotive and the dynamics of wagons are not considered for simplicity and treat them as rigid body in our simulation. But the method can be easily applied to more cars scenario.

The communication is limited due to the long distance among the cars, so the cars can only get some information of the whole train. By Theorem 6, a strongly connected communication scenario is set as Figure 4, where the locomotives in the heavy haul trains are labeled 1,2,3, and 4. The car labeled 0 describes the order imposed on the heavy haul trains. The order may come from the operator or an automatic driving system. It is easy to validate that the graph is strongly connected according to the definition in Section 2.

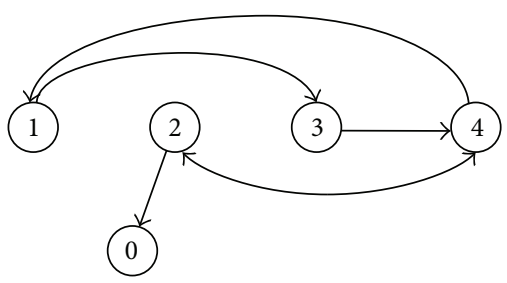

FIGURE 4: Communication topology of simulation system.

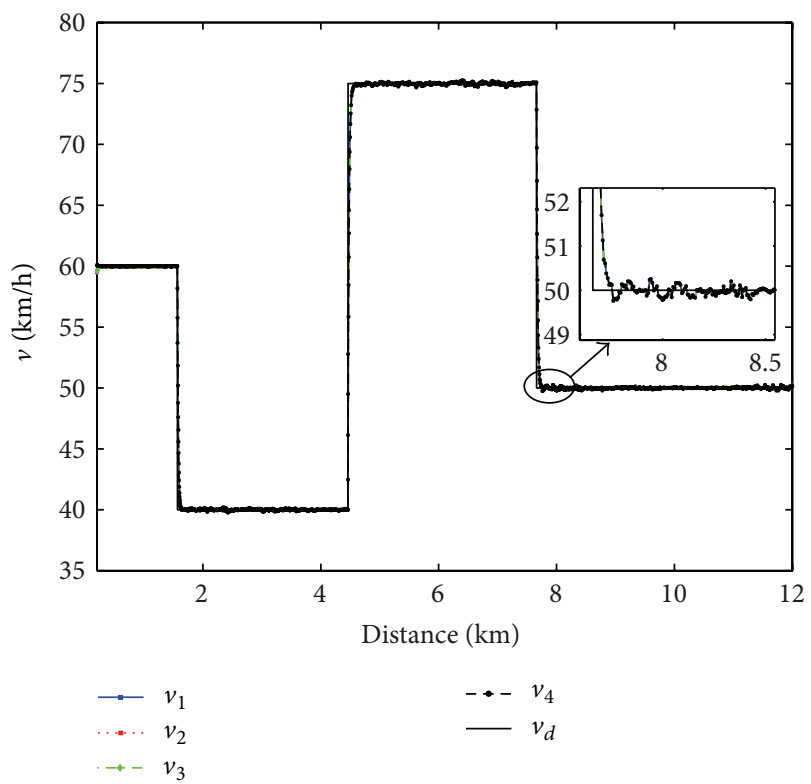

FIGURE 5: Velocity response under distributed optimal controller.

The desired speed profile $v_{0}(t)$ is given in Figure 3, and the initial speeds of the locomotives are set to be

$$
\begin{array}{llrl}
v_{1}(0) & =59.40 \mathrm{~km} / \mathrm{h}, & & v_{2}(0)=60.12 \mathrm{~km} / \mathrm{h}, \\
v_{3}(0)=59.44 \mathrm{~km} / \mathrm{h}, & v_{4}(0)=59.29 \mathrm{~km} / \mathrm{h} .
\end{array}
$$

When the distributed optimal controller is used, the velocities of the locomotives response as shown in Figure 5. The lateral axis displays the train's traveling distance during simulation. The vertical axis displays the speed response of locomotives under the controller designed in our paper. We can see that the locomotives in the heavy haul trains can track desired speed profile very well under the distributed controller with local performance index. That is because the controller aims to minimize a performance index $J_{i}$ where $\vec{\eta}_{i}$ contains a term about tracking the desired speed. When the desired speed changes, there is a transient process before the locomotives reach the new desired speed. A partial enlarged figure in Figure 5 (from $7.5 \mathrm{~km}$ to $8.5 \mathrm{~km}$ ) shows the detailed speed dynamics.

The cars' velocity deviation to the desired profile under distributed optimal controller is shown in Figure 6. The lateral axis displays the train's traveling distance during simulation. The vertical axis displays the velocity deviation to the desired profile under the controller designed in our 


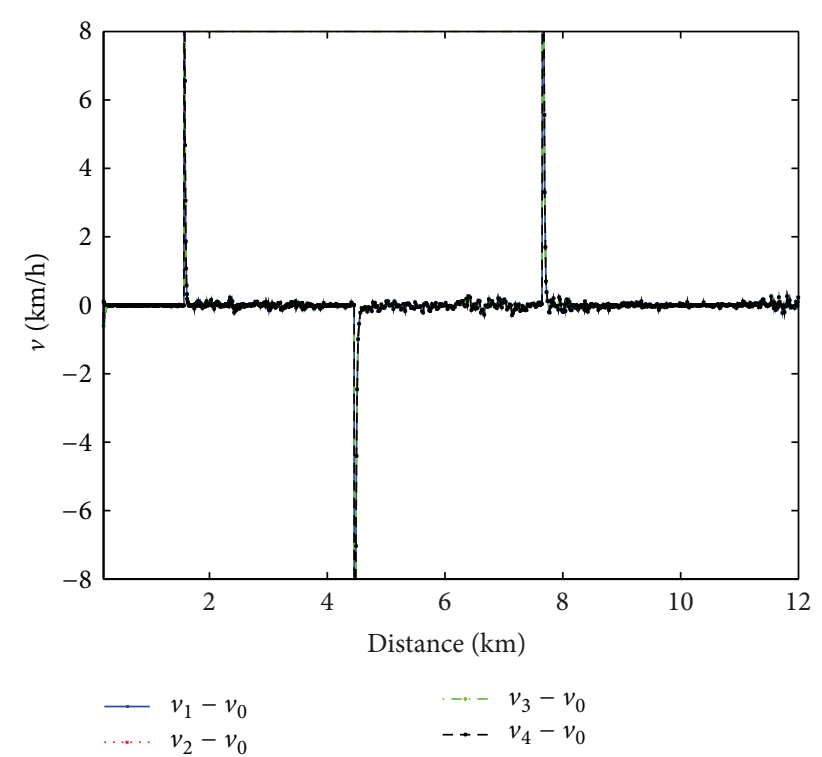

Figure 6: Velocity deviation to the desired profile under distributed optimal controller.

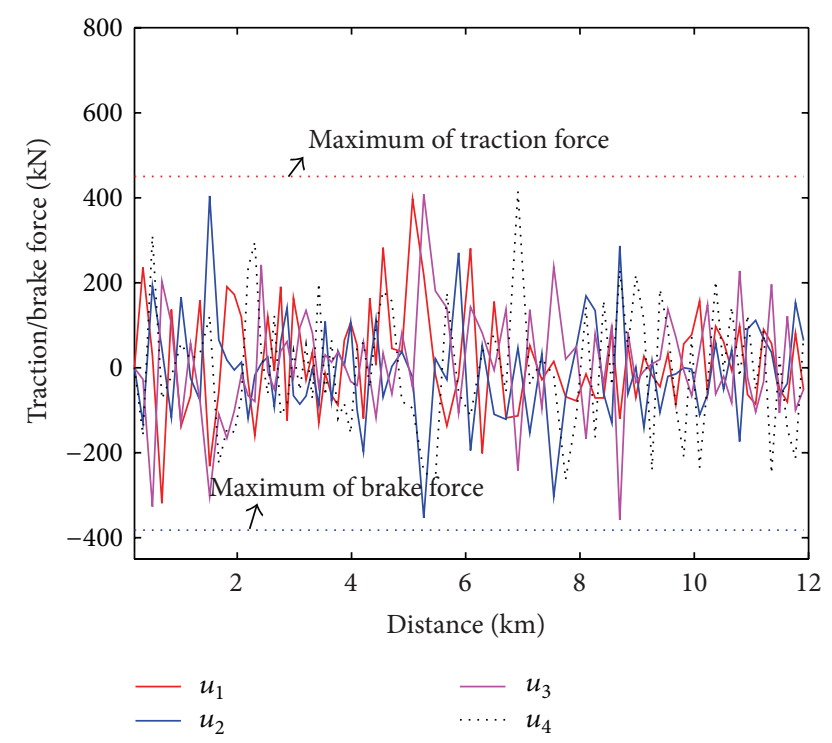

FIGURE 7: The control inputs under distributed optimal controller.

paper. We can see that the velocity deviation becomes very large when the desired speed changes but under the function of proposed controller, the deviation converges to nearly zero gradually. Because of the influence of gravitation on the ramp of railway, there is small speed fluctuation.

Figure 7 shows the resulted control inputs for each locomotive under the designed controller. The lateral axis displays the train's traveling distance during simulation. The vertical axis displays the control input including traction force and brake force under the controller designed in our paper. As shown in Table 1, the maximum of traction force is $450 \mathrm{kN}$ and the maximum of brake force is $-382 \mathrm{kN}$. It is obvious that all of the control inputs are within the normal range. The

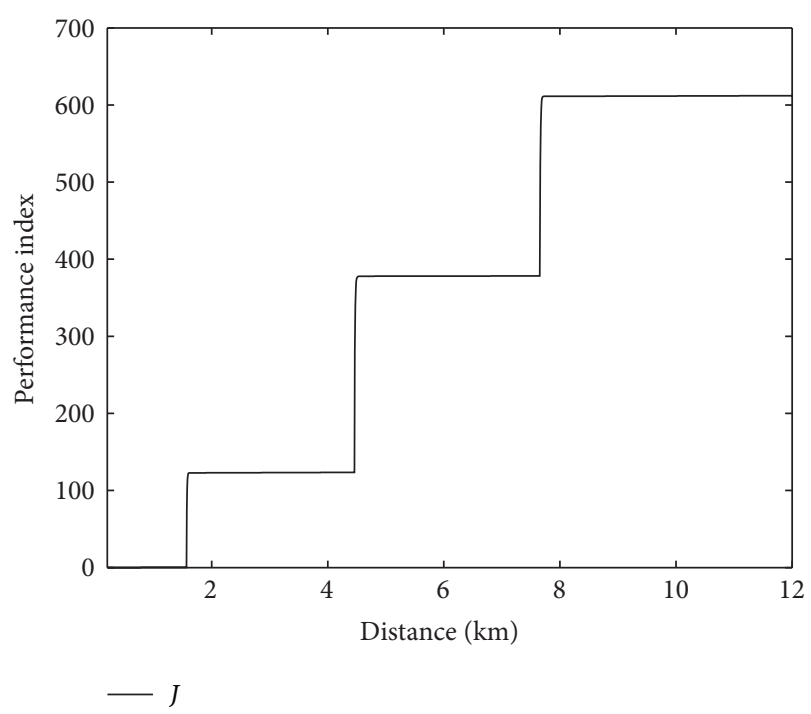

FIGURE 8: Performance index of distributed optimal controller.

ultimate value of performance index $J$ (sum of $J_{1}, J_{2}, J_{3}$, and $J_{4}$ ) of distributed optimal controller is a little bigger than 600 (Figure 8). From the definition of (6), the velocity deviation, position deviation, and control input are optimized.

\section{Conclusions}

Optimization control is an important topic in train's operation. Generally, traveling time, energy consumption, and in-train forces are considered in the performance index. Different from other types of trains, heavy haul trains are multipowered networked system with great length. In-train forces have to be considered in optimal control of heavy haul trains due to safety and cost of maintenance. Main focus has been on designing optimal controller by global information of the whole train and more recently on distributed control designed by partial information without considering the performance optimization. This paper studies the distributed optimal problem with individual performance index, which is designed by neighbors' information. The distributed optimization problem is very difficult due to couplings and conflicts among the indexes. Nonetheless, in this paper, the distributed optimization problem is formulated as a cooperative game problem. We derived the condition for achieving global Pareto optimality equilibrium in this cooperative game. An online multivariable extremum seeking algorithm is used to approximate the partial differential equation during designing the game rules. Rigorous valuation of the proposed algorithm is done against application to Daqin railway. It is revealed that the designed controller can make the train track the desired speed profile with optimization of proposed performance index.

\section{Conflict of Interests}

The authors declare that there is no conflict of interests regarding the publication of this paper. 


\section{Acknowledgment}

The authors would like to acknowledge that this work was partially supported by the National Natural Science Foundation of China (no. 61071096 and 61379111).

\section{References}

[1] M. McClanachan and C. Cole, "Current train control optimization methods with a view for application in heavy haul railways," Proceedings of the Institution of Mechanical Engineers F: Journal of Rail and Rapid Transit, vol. 226, no. 1, pp. 36-47, 2012.

[2] R. Franke, P. Terwiesch, and M. Meyer, "An algorithm for the optimal control of the driving of trains," in Proceedings of the 39th IEEE Confernce on Decision and Control, pp. 2123-2128, Sydney, Australia, December 2000.

[3] A. Albrecht, P. Howlett, P. Pudney, and X. Vu, "Optimal train control: analysis of a new local optimization principle," in Proceedings of the American Control Conference (ACC '11), pp. 1928-1933, San Francisco, Calif, USA, July 2011.

[4] Q. Gu, T. Tang, and Y.-D. Song, "A survey on energy-saving operation of railway transportation systems," Measurement and Control, vol. 43, no. 7, pp. 209-211, 2010.

[5] Y.-J. Chen, J.-A. Yu, L.-S. Zhou, and Q. Tao, "Study on the algorithm for train operation adjustment based on ordinal optimization," Advances in Mechanical Engineering, vol. 2013, Article ID 175347, 8 pages, 2013.

[6] X. Zhuan and X. Xia, "Cruise control scheduling of heavy haul trains," IEEE Transactions on Control Systems Technology, vol. 14, no. 4, pp. 757-766, 2006.

[7] M. Chou and X. Xia, "Optimal cruise control of heavy-haul trains equipped with electronically controlled pneumatic brake systems," Control Engineering Practice, vol. 15, no. 5, pp. 511-519, 2007.

[8] S. Tijs, Introduction to Game Theory, Hindustan Book Agency, New Delhi, India, 2003.

[9] N. Li and J. R. Marden, "Designing games for distributed optimization," in Proceedings of the 50th IEEE Conference on Decision and Control and European Control Conference (CDCECC '11), pp. 2434-2440, Orlando, Fla, USA, December 2011.

[10] D. Vrabie, K. G. Vamvoudakis, and F. L. Lewis, Optimal Adaptive Control and Differential Games by Reinforcement Learning Principles, Control Engineering Series, IET Press, London, UK, 2012.

[11] R. Kamalapurkar, H. Dinh, P. Walters, and W. Dixon, "Approximate optimal cooperative decentralized control for consensus in a topological network of agents with uncertain nonlinear dynamics," in Proceedings of the 1st American Control Conference (ACC '13), pp. 1320-1325, Washington, DC, USA, June 2013.

[12] J. Engwerda, "Necessary and sufficient conditions for Pareto optimal solutions of cooperative differential games," SIAM Journal on Control and Optimization, vol. 48, no. 6, pp. 38593881, 2010.

[13] P. V. Reddy and J. C. Engwerda, "Pareto optimality in infinite horizon linear quadratic differential games," Automatica, vol. 49, no. 6, pp. 1705-1714, 2013.

[14] C.-D. Yang and Y.-P. Sun, "Mixed $H_{2} / H_{\infty}$ cruise controller design for high speed train," International Journal of Control, vol. 74, no. 9, pp. 905-920, 2001.

[15] L. J. Zhang and X. T. Zhuan, "Optimal operation of heavyhaul trains equipped with electronically controlled pneumatic brake systems using model predictive control methodology," IEEE Transactions on Control Systems Technology, vol. 22, no. 1, pp. 13-22, 2014.

[16] S. Iwnicki, Handbook of Railway Vehicle Dynamics, CRC Press, London, UK, 2006.

[17] M. Mesbahi and M. Egerstedt, Graph Theoretic Methods in Multiagent Networks, Princeton University Press, New Jersey, NJ, USA, 2010.

[18] R. Olfati-Saber and R. M. Murray, "Consensus problems in networks of agents with switching topology and time-delays," IEEE Transactions on Automatic Control, vol. 49, no. 9, pp. 15201533, 2004.

[19] D. Grass, J. P. Caulkins, G. Feichtinger, and G. Tragler, Optimal Control of Nonlinear Processes: With Applications in Drugs, Corruption, and Terror, Springer, 2008.

[20] H. Li, J. Peng, W. R. Liu, Z. W. Huang, and K. C. Lin, "A Newtonbased extremum seeking MPPT method for photovoltaic systems with stochastic perturbations," International Journal of Photoenergy, vol. 2014, Article ID 938526, 13 pages, 2014.

[21] K. B. Ariyur and M. Krstić, Real-Time Optimization by Extremum-Seeking Control, Wiley-Interscience, Hoboken, NJ, USA, 2003.

[22] G. C. Walsh, "On the application of multi-parameter extremum seeking control," in Proceedings of the American Control Conference (ACC '00), pp. 411-415, Chicago, Ill, USA, June 2000. 


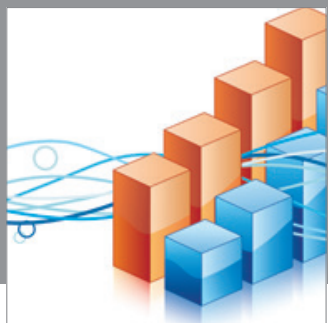

Advances in

Operations Research

mansans

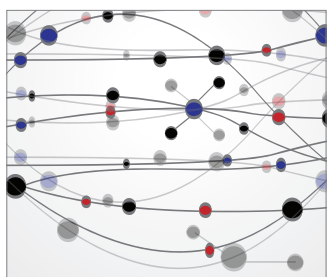

The Scientific World Journal
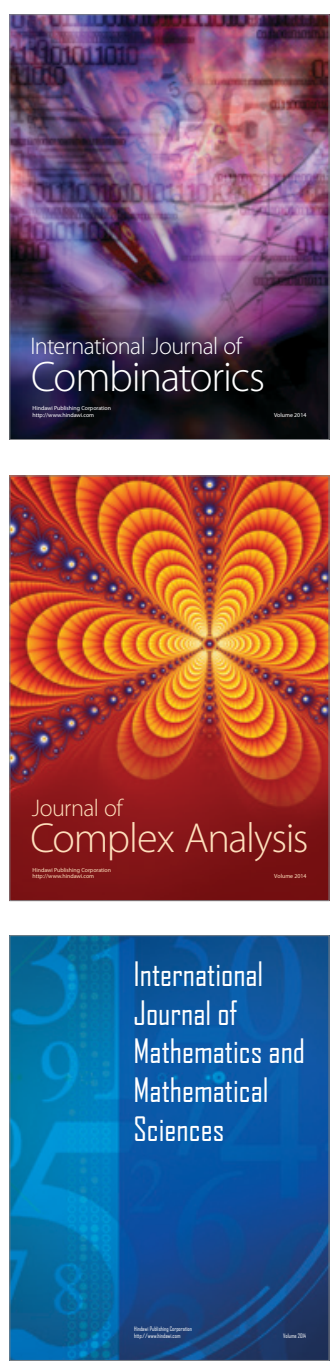
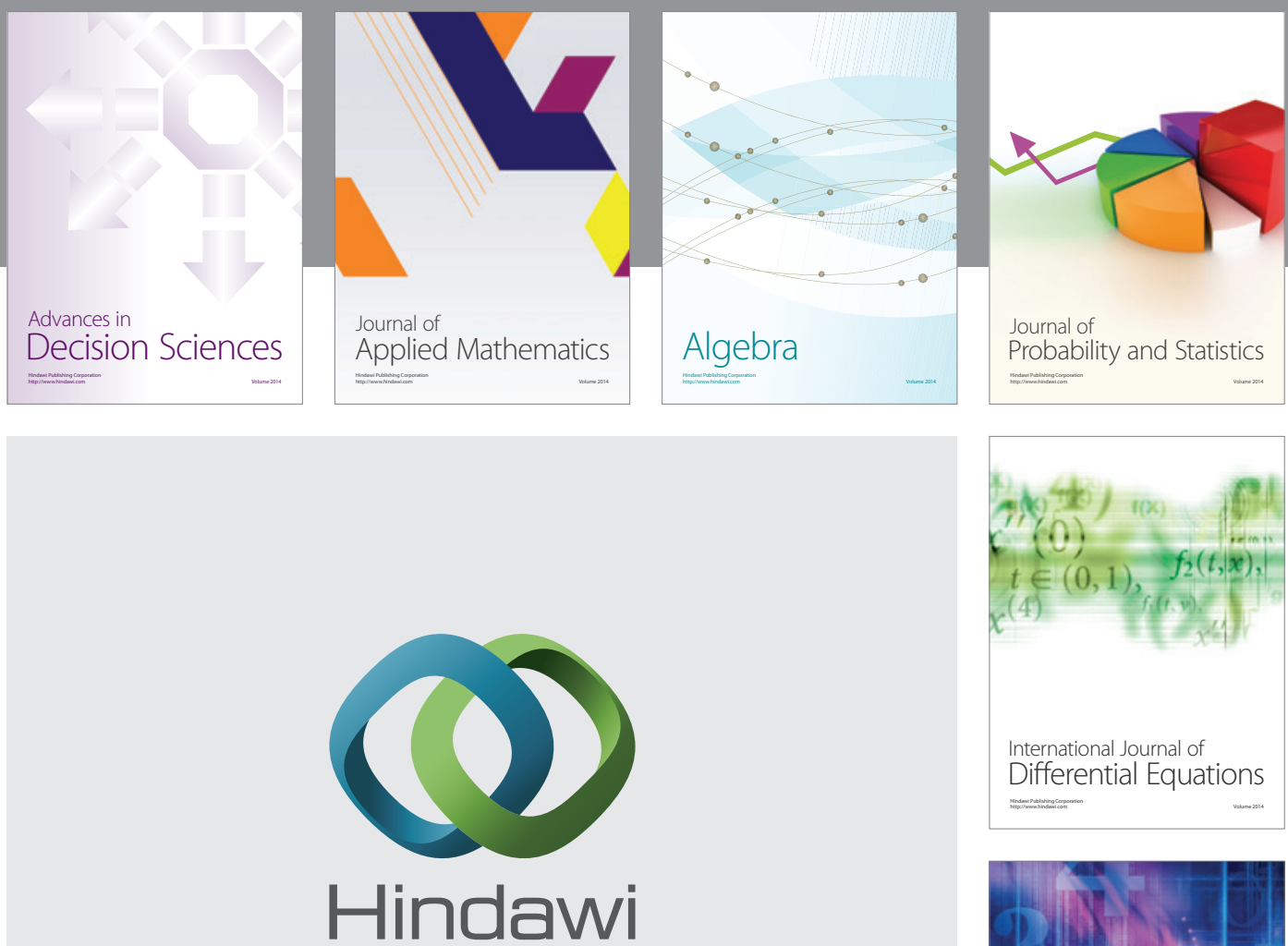

Submit your manuscripts at http://www.hindawi.com
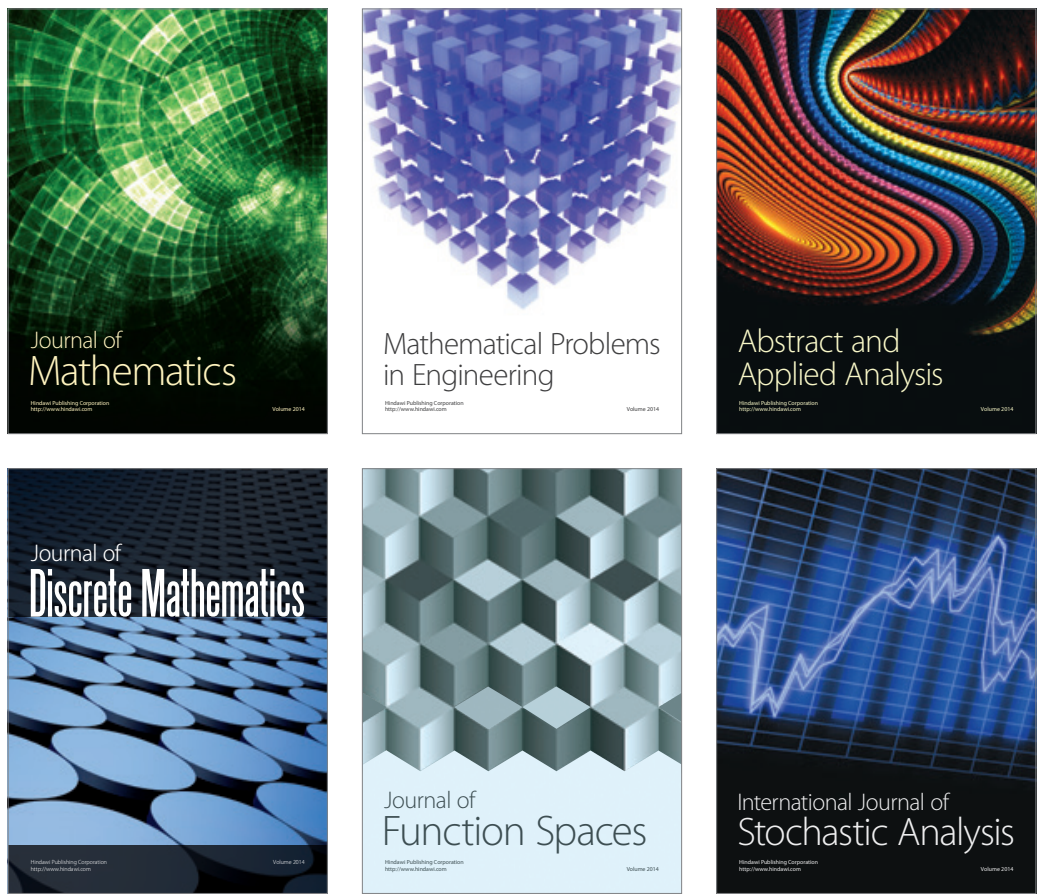

Journal of

Function Spaces

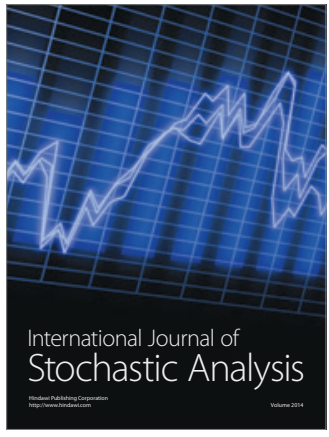

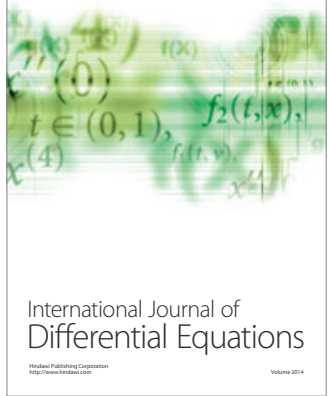
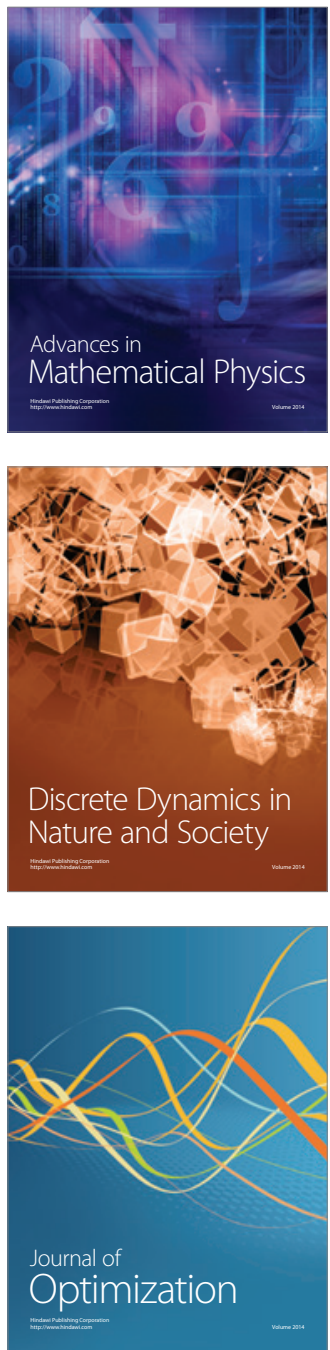